\title{
Modeling the 2017 September 10 Long Duration Gamma Ray Flare
}

\author{
James M. Ryan ${ }^{1}$ \\ University of New Hampshire \\ Durham, New Hampshire, USA \\ E-mail: james.ryan@unh.edu \\ Georgia A. de Nolfo² \\ NASA/Goddard Space Flight Center \\ Greenbelt, Maryland, USA \\ E-mail: georgia.a.denolfo@nasa.gov
}

Dale E. Gary ${ }^{3}$

New Jersey Institute of Technology

Newark, New Jersey, USA

E-mail: dale.e.garyanjit.edu

\begin{abstract}
The large flares from 2017 September 4 to 10 were significant microwave events with revealing multi-wavelength images of the flare environment. The event on September 10 was a large long-duration, gamma-ray flare (LDGRF). The event also produced a Ground Level Enhancement (GLE). Using the constraints from the microwave imaging data from the Expanded Owens Valley Solar Array (EOVSA) we interpret and model the behavior of the energetic-flare protons of September 10 as measured with the Large Area Telescope (LAT) on the Fermi mission. We do this in the context of stochastic acceleration in a large coronal bipolar structure to produce the high-energy long-duration gamma-ray emission. Our preliminary analysis suggests that the acceleration of the $\mathrm{GeV}$ protons takes place in a large structure about $1.4 R_{\odot}$ in length. The requirements for the magnetic field and turbulence in this structure will be presented.
\end{abstract}

$36^{\text {th }}$ International Cosmic Ray Conference -ICRC2019-

July 24th - August 1st, 2019

Madison, WI, U.S.A.

\section{${ }^{1}$ James M. Ryan}

(C) Copyright owned by the author(s) under the terms of the Creative Commons 


\section{Introduction and Background}

The phenomenon of Long Duration Gamma-Ray Flares (LDGRF) is a peculiar one, because the emission is singularly energetic, delayed and prolonged with respect to all other emissions emanating from the flare. Because of the delay and energies of the $\gamma$ rays, it has been postulated from the first observations that the same particles that produce ground level enhancements (GLE) also produce the $\gamma$ radiation from the Sun. However, the phenomenon is frequent, robust and repeatable. Modeling the necessary particle transport from great distances once accelerated in an IP shock is strained. Magnetic connections to the shock front are changing and transient, diffusion through the downstream region to the solar surface from distances as long as a fraction of an AU would seem to be unreliable, given the magnetic restructuring taking place behind the CME. Furthermore, one must produce a profile that is remarkably diffusive in nature, no bumps, no wiggles - a pure exponential for many hours.

Alternatively, we can investigate a diffusion solution to the particle transport and acceleration as it can take place in large coronal loops, distinct from the receding CME and shock. Modeling by Ryan and Lee (1991) shows that the trapping volumes must be large and filled with MHD turbulence to accelerate the ions via second-order Fermi acceleration and transport them diffusively to the solar photosphere where they radiate for long periods. Estimates indicate that the level of turbulence need not be excessive, provided the volume is large. Lacking is a visualization of such an active loop that could be the home and the accelerating agent for the protons. Because little else is required of the trapping loop, other than embedded turbulence, it may not readily radiate in soft $\mathrm{x}$ rays, $\mathrm{H} \alpha$ or UV. Such radiation would reveal its location, size and orientation of the loop that contains the energetic particles. However, the unique microwave observations of the 2017 September flares does reveal loops of the appropriate size and location, allowing us to model the acceleration and transport.

The events were bona fide LDGRFs with accompanying energetic ions detected in space. With the length and orientation of the loop structure measured from the microwave images. The loops are luminous in microwaves through the emission by energetic electrons and positrons. We can, thus, set constraints on the necessary embedded (and largely invisible) turbulence.

We search for a self-consistent, data-supported diffusion model of the LDGRF process without invoking a distant receding shock.

\section{Observations and Measurements}

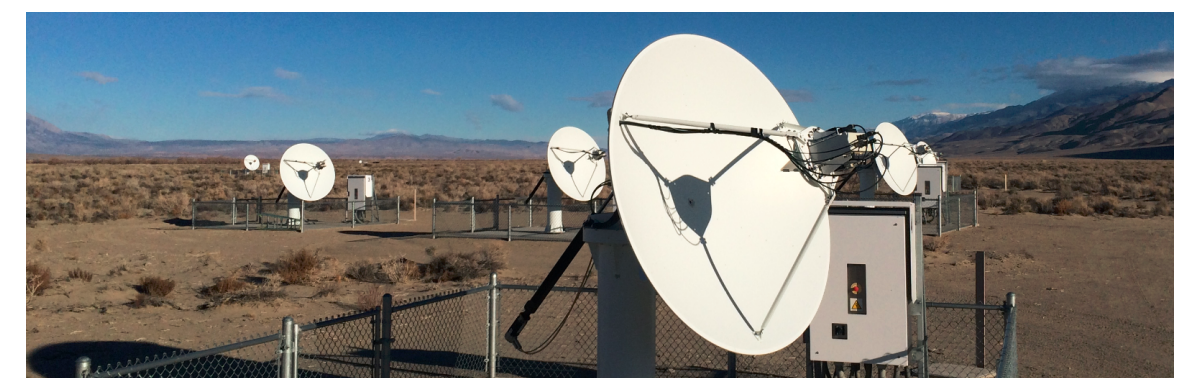

Fig. 1. Expanded Owens Valley Solar Array

The 2017 September 10 event was an unusually powerful LDGRF, emitting $>100 \mathrm{MeV} \gamma$ rays for several hours. Perhaps due to connectivity, it was a somewhat less impressive GLE and solar energetic particle (SEP) event at Earth (Bruno et al. 2018). Being on the west limb with coronal loop structures oriented with a significant north-south orientation, the event was seen in 


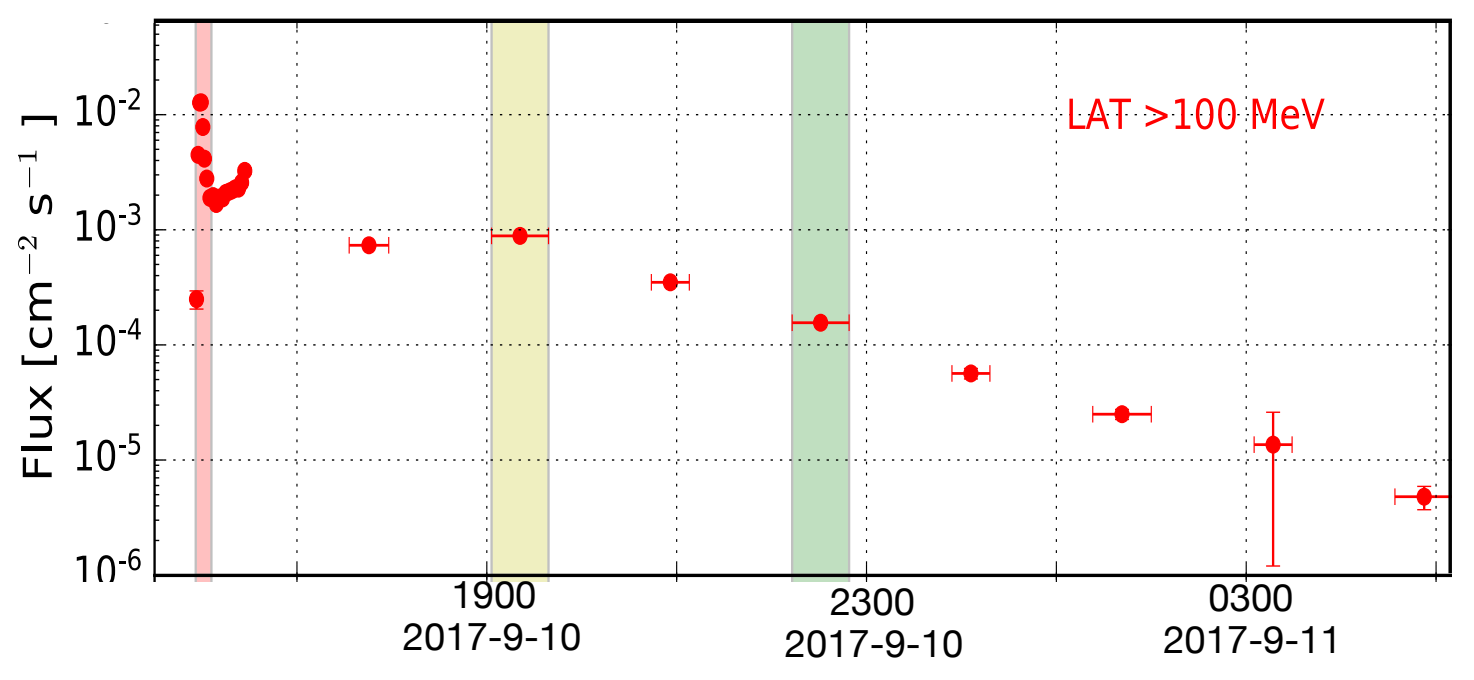

Fig. 2. Photometric $>100 \mathrm{MeV}$ profile. The colored bands represent periods of time that Omodei et al. (2018) imaged the emission. Red arrow indicates start of decay phase.

profile by EOVSA (Gary et al. 2018) (Fig. 1), allowing an examination of the dimensions of the affected volumes in the corona. Shown in Fig. 2 is the entire-event $\gamma$-ray photometry curve $>100 \mathrm{MeV}$. Fig. 3 is a snapshot of the $3.4-\mathrm{GHz}$ microwave emission at an early time in the event. It shows the legs of a loop we believe contains the energetic particles necessary for the LDGRF.

We focus on the period after 1900 UT on September 10 that exhibits a smooth photometric

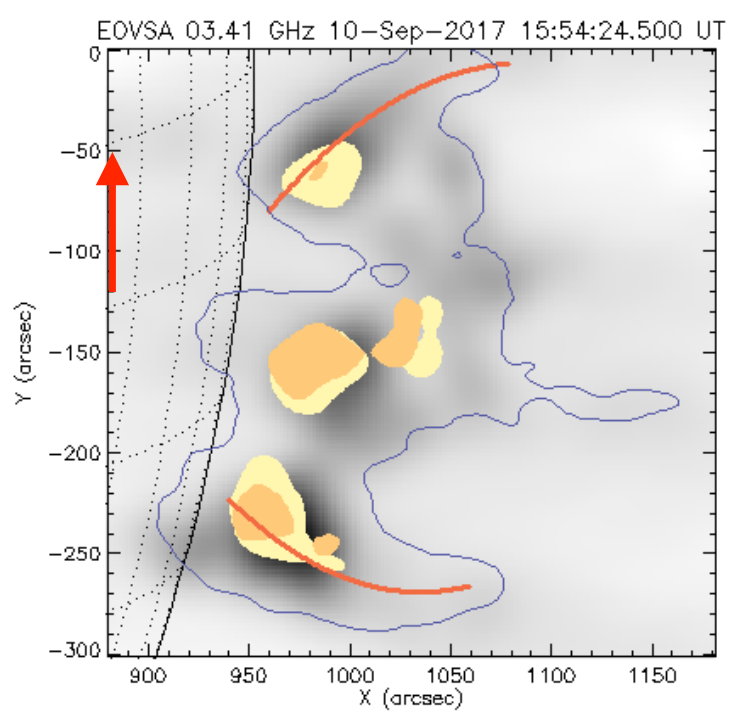

Fig. 3. $3.4 \mathrm{GHz}$ image. Red curve indicates legs of larger energized loop. West limb is shown.

exponential decay with a time constant of $\sim 6500 \mathrm{~s}$, deviating from a pure exponential by no more than $\sim 20 \%$ for over ten hours, while the deduced spectral index for the next eight hours softens from a value of 3.7 to 6 (Omodei et al. 2018), which we will return to. This time period was chosen because not only did the $\gamma$-ray event enter its exponential decay, but it is well after 
any disturbance produced by reconnections in and around the smaller internal loop and after any CME would have exited the magnetic volumes considered here, thus allowing the system to relax. There is more complicated activity leading up to the slow decay, including an impulsive phase and an intermediate recovery and fall (hidden by Earth occultation) that gives way to the $>9.5$-hour gradual feature we examine here. The onset of the long duration phase appears to have started some time earlier than 1900 UT, of order two hours earlier. The corresponding image before 1900 UT, prior to the smooth decay phase, is shown in Fig. 4 in the superposed AIA and EOVSA image at 1800 UT. It is important to note that acceleration after 1900 UT is still necessary. No form of passive trapping can support a population for that length of time without enormous (many AU) scattering mean free paths.

The microwave/AIA image in Fig. 3 is a snapshot at 1800 UT when the long duration emission is approaching its maximum, one hour before the period we are analyzing. It defines the relevant structures and their dimensions. There is an inner structure, above which, reconnection can be seen in the form of a current sheet (Fig. 4). There is also a larger structure that is highlighted in Fig. 3 with the red curves, indicating likely legs of a single larger loop. In

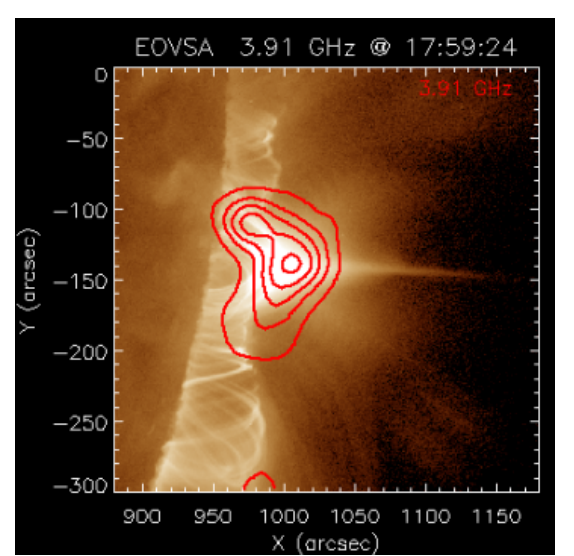

Fig. 4. Superposed $3.9 \mathrm{GHz}$ images on the 171 Å AIA image at $1800 \mathrm{UT}$.

particular, the separation of the most northern and southern lobes of this larger loop is of order $175^{\prime \prime}$ or $0.2 R_{\odot}$ in the plane normal to the observer. We take them to be the feet of a larger looplike structure. Given that the top of the loop is out of the frame, we estimate that the loop reaches a height of $0.4 R_{\odot}$ with a circular length of $1.4 R_{\odot}$, perhaps longer if it has not fully relaxed into a potential field form. We note that the microwave emission was only briefly visible in this loop, perhaps during the initial buildup of turbulence after which escape of highenergy electrons would have been inhibited to an extent that would make the emission too weak to be observable.

Shown in Fig. 4 is the AIA (171 $\AA$ ) superposed on that of the EOVSA 3.9-GHz image at 1800 UT near the peak of the gradual phase. After this time the remaining microwave and HXR activity is confined to the smaller central loop and is thermal in nature (20 MK), as is the x-ray emission (Omodei et al. 2018). No measurable non-thermal emission is detectable in the field of view of the observing instruments, other than the $100-\mathrm{MeV} \gamma$-ray emission, which is just starting its 6500-s decay. This is behavior similar to that reported by Grechnev et al. (2018) for the over-the-limb LDGRF of 2014 September 1 and earlier non-imaging observations (Chupp and Ryan 2009). 
Unfortunately, little information comes from the $\gamma$-ray image produced by Fermi at the time of the green bar in Fig. 2. The error circle of the $100 \mathrm{MeV}$ emission from LAT is large enough late in the event to capture the large loops, but this may only reflect the poorer statistics late, rather than true extended emission.

\section{Modeling}

The model in its simplest, albeit incomplete, form is a 1-d leaky box, if one only considers the long-term decay, neglecting the early time dependent aspect of the problem, i.e., the initial acceleration and transport of the particles to the footpoints, where they radiate (Ryan and Lee 1991). This initial acceleration and transport to the footpoints is seen before 1900 UT, where the emission is intensifying. The diffusion, assuming $\lambda \ll \ell$, where $\lambda$ is the particle mean free path and $\ell$ is the length of the trapping volume, governs the physical transport of the particles. It also is responsible for the acceleration of the protons via the second-order Fermi process, the time scale of the acceleration is inversely proportional to the spatial diffusion time. If we further assume that the spectral shape is stationary (which it is not, see below) then this decay in a linear box of length $\ell$ is governed only by the measured spatial diffusion time constant $\tau_{\mathrm{d}}=\ell^{2} / \pi^{2} \kappa$, where $\kappa$ is the inferred or measured spatial diffusion coefficient, assumed to be momentum or energy independent. The quantity $\kappa$, however, does not fully capture the observed decay of the particle distribution. The value of $\kappa$ should be considered an upper limit, because acceleration continues during the transport and the precipitation process. That said, we take the acceleration rate with respect to the loss rate to be stabilized and that the spectral shape of the ions above the $\gamma$-ray production threshold $(\sim 300 \mathrm{MeV})$ is steady. The softening of the spectrum with time, however, belies this assumption to some degree, but probably not too much, given that the spectrum is still well represented by a power law. In summary, if one knows both $\ell$ and $\tau_{\mathrm{d}}$, one can deduce $\kappa$, the diffusion coefficient. In our case, neglecting acceleration, the gradual phase time constant is $\leqslant 6500 \mathrm{~s}$ that we set equal to $\kappa$. From this, quasi-linear theory can be used to estimate some plasma properties within the trap, such as $\delta B / B$, and from that the spectrum-integrated wave-energy density (e.g., Lee, 1982).

A 6500 -s precipitation of particles to the footpoint in a loop of length $1.4 R \odot$ requires a diffusion coefficient of $1.4 \times 10^{17} \mathrm{~cm}^{2}-\mathrm{s}^{-1}$, corresponding to $\lambda \sim 200 \mathrm{~km}$, consistent with the diffusion approximation.

To see how much wave intensity we need, we normalize the wave power spectrum to the intensity at the resonant wave number in a $1-G$ field. From there we extend the $k^{-5 / 3}$ Kolmogorov form to a $k$ value representative of the loop cross section diameter that we take to be $\ell / 10$, where it is flat to the origin. This in turn implies a wave field energy of $0.7 \mathrm{ergs}^{-\mathrm{cm}^{-3}}$, which exceeds the ambient magnetic field energy at $1 \mathrm{G}\left(0.04\right.$ ergs- $\left.\mathrm{cm}^{-3}\right)$, what we might expect at an altitude of $0.4 R \odot$. However, no such problem exists farther down the legs of the loop, where $B$ is much greater. For example, when $B \sim 10 \mathrm{G}$, only $18 \%$ of the ambient $B$ energy need be in the form of waves. The situation improves rapidly with increasing $B$ in a dipolar field.

\section{Discussion}

Are we accelerating particles? An acceleration time scale $\tau_{\mathrm{a}}\left(=9 \kappa / V_{\mathrm{A}}{ }^{2}\right)$ (Schlickeiser 1986) can be computed, where $V_{\mathrm{A}}$ is the Alfvén speed. For the acceleration time scale to equal the diffusion time scale, one needs an Alfvén speed $>140 \mathrm{~km}-\mathrm{s}^{-1}$, a modest requirement. Greater speeds shorten $\tau_{\mathrm{a}}$. 
This simple analysis reveals several things:

i. With unprecedented microwave imaging, we have been able to put some realistic numbers on coronal traps that can produce protons of sufficient energy to power an LDGRF.

ii. Accompanying images and data from SDO, RHESSI and LAT allows us to quantitatively examine the energetic particle environment.

iii. We see that diffusion in a static loop can successfully both impede the transport of particles, while accelerating them to the requisite energy.

However, this new information highlights shortcomings of the model.

i. Grechnev et al. (2018) concluded that a shock passage seeds the large loop, in which the diffusion process occurs. This event is similar, in that, the reconnection site associated with the central HXR, AIA and $\mu$-wave image gives rise to a breakout process (Karpen et al. 2018), producing a shock that likely accelerates particles to modest energy at low altitudes. However, after three hours, the region behind the blob will likely re-configure into a more dipole like structure. This would leave behind a large loop with seed particles, with those particles being unrelated to those in the impulsive phase, reminiscent of Hudson's lasso picture (Hudson 2018).

ii. The model is too simple as is. For large loops we must incorporate the inhomogeneity of magnetic field.

iii. In regions where $B$ is too small to support the necessary wave field, the containment of the wave energy must be included, perhaps similar to that discussed by Hollweg (1984) where waves in loops are reflected off gradients and discontinuities in the "index of refraction."

iv. Investigate self-generated waves produced by the low-energy protons that resonate with higher energy protons, producing a non-Kolmogorov spectrum, similar to that computed by Lee (1982).

v. One must include a momentum-dependent diffusion coefficient that will produce a varying power law spectral index.

\section{Conclusions}

Conceptually and qualitatively, a coronal trap, with spatial and momentum diffusion governing the precipitation of high-energy particles, can re-produce LDGRF behavior witnessed many times since 1982 (Chupp et al. 1983). The diffusive behavior produces a "perfect" exponential decay, difficult to achieve by other processes. Realistic numbers obtained from new measurements of an actual environment clears the way for future modeling efforts.

\section{Acknowledgments}

We thank Dr. M.A. Lee for helpful discussions.

This work was supported by NASA grant NNX16AL66G (UNH and GSFC). This research is also supported by NSF grant AST-1615807 andNASA grants 80NSSC18K1128 and 80NSSC19K0068 to New Jersey Institute of Technology. 


\section{References}

1. Bruno, A. et al. 2019, Spectral Analysis of the September 2017 Solar Energetic Particle Events, Space Weather, 17, 418.

2. Chupp, E.L. and J.M. Ryan 2009, High energy neutron and pion-decay gamma-ray emissions from solar flares, Res. in Astron. and Astrophys., 9.

3. Chupp, E.L. et al. 1987, High energy neutron and pion-decay gamma-ray emissions from solar flares, Astrophys. J., 318.

4. Gary et al., 2018, Microwave and Hard X-Ray Observations of the 2017 September 10 Solar Limb Flare, Astrophys. J., 863, 83.

5. Grechnev et al. 2018, Radio, Hard X-Ray, and Gamma-Ray Emissions Associated with a Far-Side Solar Event, Solar Phys., 293, 133.

6. Hollweg, J. 1984, Alfvénic resonant cavities in the solar atmosphere: Simple aspects, Astrophys. J., 91, 269.

7. Hudson, H. 2018, The Relationship between Long-Duration Gamma-Ray Flares and Solar Cosmic Rays, Space Weather of the Heliosphere: Processes and Forecasts, Proceedings of IAU Symp. No. 335, 49.

8. Karpen, J. et al., 2019, New Insights into the 10 September 2017 Mega-Eruption, Bull. Am. Astron. Soc., 51.

9. Lee, M.A. 1982, Coupled hydromagnetic wave excitation and ion acceleration upstream of the earth's bow shock, J. Geophys. Res., 87.

10.Omodei et al. 2018, Fermi-LAT Observations of the 2017 September 10 Solar Flare, Astrophys. J. Lett., 865, L7.

11.Ryan, J.M. and M.A. Lee, 1991, On the Transport and Acceleration of Solar Flare Particles in a Coronal Loop, Astrophys. J., 368.

12.Schlickeiser, R. 1986, Stochastic Particle Acceleration in Cosmic Objects, in Cosmic Radiation in Contemporary Astrophysics, Reidel. 